\title{
Targeting Histone Deacetylases in Malignant Melanoma: A Future Therapeutic Agent or Just Great Expectations?
}

\author{
NIKOLAOS GARMPIS ${ }^{1 *}$, CHRISTOS DAMASKOS ${ }^{1,2^{*}}$, ANNA GARMPI $^{3 *}$, \\ DIMITRIOS DIMITROULIS ${ }^{1 *}$, ELEFTHERIOS SPARTALIS ${ }^{2}$, GEORGIOS-ANTONIOS MARGONIS ${ }^{4}$, \\ DIMITRIOS SCHIZAS ${ }^{5}$, IRINI DESKOU ${ }^{5}$, CHRYSOULA DOULA $^{6}$, ELENI MAGKOUTI ${ }^{7}$, \\ NIKOLAOS ANDREATOS ${ }^{4}$, EFSTATHIOS A. ANTONIOU ${ }^{1}$, AFRODITI NONNI ${ }^{8}$, \\ KONSTANTINOS KONTZOGLOU ${ }^{1}$ and DIMITRIOS MANTAS ${ }^{1}$ \\ ${ }^{1}$ Second Department of Propedeutic Surgery, Laiko General Hospital, Medical School, \\ National and Kapodistrian University of Athens, Athens, Greece; \\ ${ }^{2}$ N.S. Christeas Laboratory of Experimental Surgery and Surgical Research, Medical School, \\ National and Kapodistrian University of Athens, Athens, Greece; \\ ${ }^{3}$ Internal Medicine Department, Laiko General Hospital, University of Athens Medical School, Athens, Greece; \\ ${ }^{4}$ Department of Surgery, The Johns Hopkins University School of Medicine, Baltimore, MD, U.S.A.; \\ ${ }^{5}$ First Department of Surgery, Laiko General Hospital, Medical School, \\ National and Kapodistrian University of Athens, Athens, Greece; \\ ${ }^{6}$ Plastic Surgery Department and Burn Unit, Nicosia General Hospital, Nicosia, Cyprus; \\ ${ }^{7}$ Department of Neurology, Agia Sofia General Children's Hospital, Athens, Greece; \\ ${ }^{8}$ First Department of Pathology, School of Medicine, \\ National and Kapodistrian University of Athens, Athens, Greece
}

\begin{abstract}
Background/Aim: Malignant melanoma is the most aggressive type of skin cancer, with increasing frequency and mortality. Melanoma is characterized by rapid proliferation and metastases. Malignant transformation of normal melanocytes is associated with imbalance between oncogenes' action and tumor suppressor genes. Mutations or inactivation of these genes plays an important role in the pathogenesis of malignant melanoma. Many target-specific agents improved progression-free survival but unfortunately metastatic melanoma remains incurable, so new therapeutic
\end{abstract}

This article is freely accessible online.

*These Authors contributed equally to this study.

Correspondence to: Dr. Nikolaos Garmpis, Second Department of Propedeutic Surgery, Laiko General Hospital, National and Kapodistrian University of Athens, Medical School, 17 Agiou Thoma Street, 11527, Athens, Greece. Tel: +30 6976291403, Fax: +302132061772, e-mail: nikosg22@hotmail.com

Key Words: Histone, deacetylase, HDAC, inhibitor, malignant, melanoma, targeted, epigenetics, review. strategies are needed. The balance of histones' acetylation affects cell cycle progression, differentiation and apoptosis. Histone deacetylases (HDAC) are associated with different types of cancer. Histone deacetylase inhibitors (HDACI) are enzymes that inhibit the action of HDAC, resulting in block of tumor cell proliferation. A small number of these enzymes has been studied regarding their anticancer effects in melanoma. The purpose of this article was to review the therapeutic effect of HDACI against malignant melanoma, enlightening the molecular mechanisms of their action. Materials and Methods: The MEDLINE database was used. The keywords/ phrases were; HDACI, melanoma, targeted therapies for melanoma. Our final conclusions were based on studies that didn't refer solely to melanoma due to their wider experimental data. Thirty-two articles were selected from the total number of the search's results. Only English articles published until March 2017 were used. Results: Molecules, such as valproid acid (VPA), LBH589, LAQ824 (dacinostat), vorinostat, tubacin, sirtinol and tx-527, suberoyl bishydroxamic acid (SBHA), depsipeptide and Trichostatin A (TSA) have shown promising antineoplastic effects against melanoma. Conclusion: HDACI represent a promising agent for targeted therapy. More trials are required. 
Malignant melanoma is the most aggressive type of skin cancer. It arises from melanocytes and spreads via the bloodstream and the lymphatic system. It emerges in the skin and the mucous membranes of the mouth and genitals (1). Among melanoma cases, $90 \%$ afford the skin and $10 \%$ the eyeball, the brain meninges, the mucous membrane of the oral cavity and the genitalia (2). The frequency of melanoma is constantly increasing worldwide and mortality is rising faster than any other form of cancer (3).

Melanoma is characterized by rapid proliferation and numerous metastases. Malignant transformation of the normal melanocytes is associated with the imbalance between the action of oncogenes and tumor suppressor genes. Mutations or inactivation of these genes plays an important role in the pathogenesis of the malignant melanoma (1).

In recent years many target-specific agents [i.e. BRAF inhibitors, Histone deacetylase inhibitors (HDACI)] have been developed and have improved progression-free survival but unfortunately metastatic melanoma is still incurable. A variety of factors have been associated with resistance to therapy such as tumor cell alterations during tumor progression and the microenvironment including chemokines (4).

Recent studies have shown that BRAF mutations affect melanoma cell survival through the MAPK pathway. Especially MAPK activation leads to transcriptional changes of the DNA which have an important role in melanoma cell differentiation and proliferation. Almost $40 \%$ of cutaneous melanoma patients have tumors with BRAF mutations which activate the MAPK pathway through phosphorylation that activates $M E K$ and $E R K$ genes. Phosphorylation of these genes leads to enhanced cell proliferation and survival signals (5).

Histone deacetylase (HDAC) proteins get involved in the malignant transformation by affecting the chromatin structure and the transcription of several genes that are associated with the cell cycle. HDACI are pharmacologic compounds that interfere with the deacetylation process mediated by HDAC, leading to a global increase of histone acetylation. HDACI represent promising therapeutic agents because they can affect the survival of tumor cells and their environment (4).

\section{HDAC Classification}

HDAC comprise of 18 different substances subdivided into four classes: class I consists of HDAC-1, $-2,-3$ and -8 and are located in the cell nucleus. Class II consists of HDAC$4,-5,-6,-7,-9$ and -10 that are characterized by exonuclear and nuclear localization. Class III includes sirtuins (SRT-17 genes) and Class IV consists of only HDAC-11 which has characteristics of both Class I and II. Classes I, II and IV of HDAC require a zinc $\left(\mathrm{Zn}^{+}\right)$ion to transfer acetyl groups
Table I. Classification of histone deacetylases.

\begin{tabular}{lcccc}
\hline Class I & \multirow{2}{*}{ Class II } & & Class III & Class IV \\
\hline HDAC-1 & \multirow{2}{*}{ IIa } & HDAC-4 & SIRT-1 & HDAC-11 \\
HDAC-2 & & HDAC-5 & SIRT-2 & \\
HDAC-3 & & HDAC-7 & SIRT-3 & \\
HDAC-8 & & HDAC-9 & SIRT-4 & \\
& IIb & HDAC-6 & SIRT-5 & \\
& & HDAC-10 & SIRT-6 & \\
& & & SIRT-7 & \\
\hline \multicolumn{2}{c}{$\mathrm{Zn}^{+}$-dependent } & NAD & \\
\hline
\end{tabular}

from their substrates. Sirtuins have no similarities with the other three groups of HDAC and require a nicotinamide adenine dinucleotide $\left(\mathrm{NAD}^{+}\right)$ion for their catalytic activity (6) (Table I).

\section{HDAC Mechanism of Action}

The role of HDAC is opposite to the activity of histone acetyltransferases (HAT). The balance between the action of HDAC and HAT define the level of acetylation of the histones. HDAC catalyze the removal of the acetyl group on lysine residues of proteins and nucleosome histones $\mathrm{H} 2 \mathrm{~A}$, $\mathrm{H} 2 \mathrm{~B}, \mathrm{H} 3$ and $\mathrm{H} 4$ are some of the substrates of HDAC. The balance of acetylation of histones, regulates the chromatin structure and as a consequence the transcription of many genes. Acetylation of lysine residues neutralizes the histones and leads to a less condensed chromatin structure by increasing the space between nucleosome and the DNA wrapped around it (Figure 1). As a result of this change to the chromatin structure, transcription factors can get combined with the DNA in such a way that it can activate the transcription of many genes that get involved in the control of the cell cycle progression, differentiation and apoptosis (5, 6). Moreover, it has been shown that other acetylated proteins are substrates of HDAC, including NF-YA, p53 and GATA-1 (6). HDAC have been associated with different types of cancer. Some of them include over-expression of HDAC-1 and -5 associated with gastric cancer, HDAC-2 and -3 have been found in colorectal cancer and SIRT- 8 is over-expressed in thyroid cancer $(6,7)$. In addition, HDAC-1, -2 and -3 have been associated with high levels of activated NF-kB which result in poor prognosis in patients with pancreatic cancer (7).

\section{Anti-cancer Action of HDACI in Melanoma}

HDACI are enzymes that inhibit the action of HDAC causing hyperacetylation of their substrates (Figure 1). Extensive research has shown that HDACI can block tumor cell 


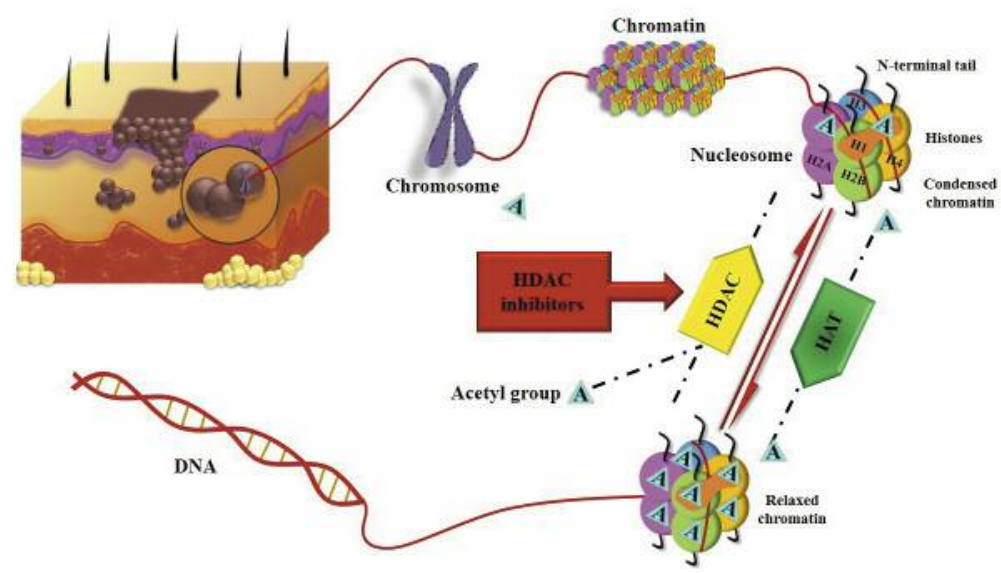

Figure 1. Mechanism of action of histone deacetylase inhibitors (HDACI) as targeted therapy against malignant melanoma. Histone acetyl transferases (HAT) catalyze lysine acetylation; Histone deacetylases (HDAC) remove acetyl groups. HAT: Histone acetyltransferases; HDAC: histone deacetylases.

proliferation and differentiation by affecting the balance of histone acetylation. There are four main classes of HDACI that differ regarding their structure, hydroxamic acid, cyclic tetrapeptide, benzamide and aliphatic acid (6). Although there is a big variety of these enzymes, a small number has been studied about their anticancer action in melanoma.

Extensive research has shown that acetylation of many genes takes place during melanogenesis and this reaction contributes to the down-regulation of the tumor suppressor genes such as p14ARF and p16INK4a (6). Apart from the histones, HSP90, p53, NF-kB and the subunit of p65 are also targets of HDAC proteins.

Studies on the HDAC function, have shown that hyperacetylation of protein HSP90 following HDAC inhibition causes: a) the degradation of C-RAF and Akt signaling molecules which have an important role as mediators of growth and $b$ ) degradation of the upstream receptor tyrosine kinases ERBB1 and ERBB2 that is associated with microtubule affinity regulating kinase (MARK) inhibitor resistance.

In addition, alteration of p53 activity and activation of NF-kB may alter melanoma resistance to therapy and may also lead in differentiation in cytokine production, transcription of anti-apoptotic protein and immune response $(1,6)$. Table II summarizes the major actions of HDACI against malignant melanoma.

$V P A$. Induction of apoptosis is a significant effect of valproic acid (VPA), an extensively studied HDACI. VPA increases the activity of two of the caspase-3, by inhibiting the activity of two of the four classes of HDAC, I and II, except for HDAC- 9 and -11 which are activated by VPA (1). Caspase3 belongs to cysteine proteases ICE and participates in the apoptosis procedure. VPA also inhibits angiogenesis and metastasis inducing the cell cycle arrest in G1 phase. This action of valproic acid is achieved with up-regulation of p16 protein, a cell-cycle inhibitor (2). It has also been shown that pan-HDACI enhancing apoptosis in malignancy with upregulation of BIM, BAX and BIK, proteins with apoptotic action and with down-regulation of Bcl-XL and XIAP which have anti-apoptotic action (8).

HDAC-9 and -11 are activated by VPA (1). Caspase- 3 is involved in the apoptosis and it can be activated by depsipeptide and SAHA (9, 10). Besides VPA inhibits angiogenesis and metastasis inducing the cell cycle arrest in G1 phase. This action of valproic acid is achieved with upregulation of the p16 protein, a cell-cycle inhibitor (2). It has also been shown that pan-HDACI enhance apoptosis in malignancy with up-regulation of BIM, BAX and BIK, proteins with apoptotic action and with down-regulation of Bcl-XL and XIAP which have anti-apoptotic action $(8,10-12)$.

$L B H 589$. It has been found that low nanomolar concentrations of LBH589 inhibit the growth of all melanoma cell lines tested, but not normal melanocytes. This inhibition is characterized by increased apoptosis as well as a G1 cell cycle arrest. In addition, LBH589 augments the expression of MHC and co-stimulatory molecules on melanoma cells leading to an increased ability to activate antigen specific T-cells. In vivo, LBH589 treatment of melanoma-bearing mice results in a significant increase in survival (13).

LAQ824. The HDACI LAQ824 has antitumor activity in melanoma tumors, but the degree of induced apoptosis may vary. It induces the expression of $\mathrm{p} 21$, increases production of reactive oxygen species and activates the caspase cascade (14). When combined with retinoids, therapeutic results are promising. 
Table II. Major actions of histone deacetylase inhibitors against malignant melanoma.

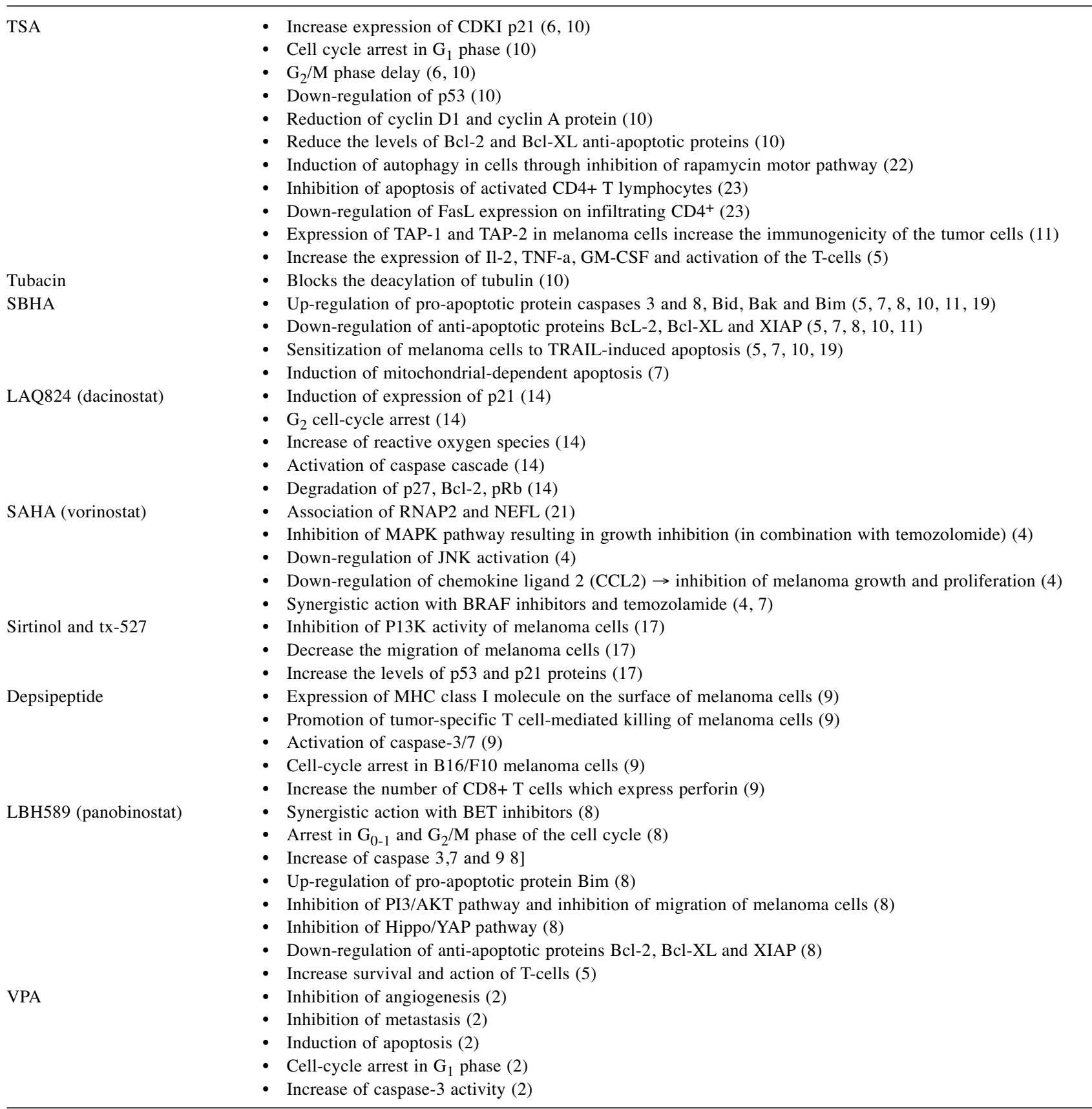

TSA: Trichostatin A; CDKI: cyclin-dependent kinase inhibitor; SBHA: suberic bishydroxamate; SAHA: suberoylanilidehydroxamic acid; NELF: negative elongation factor; CCL 2: chemokine (C-C motif) ligand 2; VPA: valproic acid.

Vorinostat. The safety and efficacy of the hydroxamate vorinostat (SAHA) were assessed in combination with the proteasome inhibitor marizomib in melanoma, pancreatic cancer and lung cancer in a phase I study. The efficacy of both agents was assessed in vitro in tumor cell lines revealing a marked synergy in terms of growth inhibition and induction of apoptosis (15).

Tubacin. HDAC-6 inhibition with tubacin results in the accumulation of acetylated $\alpha$-tubulin, but not acetylated 
histones in normal human foreskin fibroblast (HFS) cells and transformed human prostate cancer (LNCaP) cells. Tubacin reduces the rate of growth of transformed and, to a lesser extent, normal cells, without loss of cell viability (16).

Sirtinol and tx-527. SIRT- 1 is a member of the class III HDAC known as the Sirtuins, which depend on nicotinamide adenine dinucleotide (NADC) as a substrate. Wilking et al. (17), studied the action of SIRT-1 inhibitors and found that the inhibition of SRT1 decreases the phosphorylation of PIP2 in PIP3 by inhibiting PI3K so the cell migration is limited (13). SIRT-1 inhibitors can increase the levels of p53 and p21, two proteins which are important for the cell-cycle arrest to inhibit the proliferation of tumor cells (17). The role of SIRT-1 in melanoma is beginning to be unraveled only recently and SIRT-1 inhibition seem to affect p53 protein levels in wild-type p53 melanoma cells, while several other pathways may be affected by SIRT-1 inhibition, including a possible role in cell-cycle regulation. Other studies have shown that the combination of pan-HDACI SAHA and termozolamide might have a therapeutic advantage in melanoma treatment by causing apoptosis in tumor cells. The combination of these two drugs especially, is responsible for down-regulation of chemokine (C-C motif) ligand 2 (CCL 2 ), which is associated with melanoma growth and proliferation (4).

SBHA. The new HDACI suberic bishydroxamate (SBHA), is responsible for its effect on a panel of human melanoma cell lines. It induces varying degrees of apoptosis in the melanoma lines but not in melanocytes and fibroblasts. Induction of apoptosis is caspase dependent and associated with induction of changes in mitochondrial membrane permeability, which could be inhibited by overexpression of Bcl-2. SBHA down-regulates several key antiapoptotic proteins including $\mathrm{X}$-linked inhibitor of apoptosis and the Bcl-2 family proteins, Bcl-XL and Mcl-1. In contrast, it induces up regulation of the $\mathrm{Bcl}-2$ family proapoptotic proteins, Bim, Bax, and Bak (18). In the study of Gillespie et al., TRAIL and SBHA synergistically induce apoptosis of melanoma cells as revealed by quantitative analysis using the normalized isobologram method. This is supported by enhanced activation of caspase- 3 and cleavage of its substrates, PARP and ICAD (19).

Despipeptide. Another study approved that in the presence of despipeptide, a well-known HDACI, B16/F10 melanoma cells induced apoptotic cell death after Fas-FasL crosslinking, something that does not happen with Fas crosslinking alone $(9,20)$. Besides, depsipeptide can increase the perforin expressing CTLs and perforin in conjunction with granzyme induces an apoptotic cascade in melanoma cells (9). Another study has proved that HDACI LAQ 824 in combination with retinoic acid can increase the production of ROS, leading to the activation of caspase cascade and degradation of $\mathrm{Bcl}-2$ anti-apoptotic protein in melanoma cells (14). Antineoplastic action of HDACI is related with the prevention of transcription $(9,21)$. Greer et al. showed that HDACI can promote the connection between RNAP2 and negative elongation factor (NELF), which blocks elongation by preventing the action of RNAP2. The same research revealed that HDACI can prevent the transcription of many genes with an additional mechanism: HDACI gets associated with the redistribution of bromodomaincontaining protein 4 (BRD-4) which recruits the positivetranscription elongation factor $\mathrm{b}$ (P-T EFb). P-T EFb has an important role in the transcription of many genes because it modifies RNAP2 and other factors required for overcoming elongation block (21).

Trichostatin A (TSA). Trichostatin is a "non-specific" HDACI. It increases expression of the cyclin-dependent kinase inhibitor (CDKI) p21, while it down-regulates p53. It causes reduction of cyclin D1 and cyclin A protein (10) and reduces the levels of $\mathrm{Bcl}-2$ and $\mathrm{Bcl}-\mathrm{XL}$ anti-apoptotic proteins. It also increases the expression of Il-2, TNF-a, GMCSF and the activation of T-cells. Moreover, TSA induces autophagy in cells through inhibition of rapamycin motor pathway (22) and inhibits apoptosis of activated $\mathrm{CD} 4^{+} \mathrm{T}$ lymphocytes (23). Butyrate as well as trichostatin A inhibited the cell growth mainly by arresting the cell cycle and the cell invasion was inhibited by butyrate and trichostatin A. At low doses, sodium butyrate and trichostatin induced a $G_{1}$ cellcycle block in melanoma cells (23).

\section{Discussion}

It is speculated that HDACI sensitize melanoma cells to immunotherapy. Malignant melanomas in situ contain a high level of HDAC-1, 2 and malignant melanoma cells overexpress HDAC-1, -2, -3 compared with non-cancer cells. Furthermore, pharmacologic inhibition of class I HDAC sensitizes malignant melanoma cells to apoptosis following exposure to alkylating agents, while not affecting primary melanocytes (24-26).

West et al. (27) showed that the immune system is absolutely necessary for the anticancer effect of HDACI ( 9 , $11,24,28)$. HDACI distribute to the up-regulation of natural killer NK1-cell activating ligands MHC class I and II, which are responsible for antigen presentation on the surface of cancer cells (22). HDACI can sensitize malignant cells to the antineoplastic effect of IFN- $\gamma$ by increasing the number of IFN- $\gamma$ receptors (IFNGR1) on the surface of cancer cells. The same group approved that B cells have a critical role in the anticancer effect of HDACI by producing IFN- $\gamma(27)$. 
Recent micro-array studies revealed that depsipeptide a well-known HDACI, increases gp100 expression on the surface of melanoma cells. This HDAC class I molecule is released from the endoplasmic reticulum reaches the cell surface and this up-regulation enhances the immunological recognition. The same study showed that depsipeptide can increase the action of T-cells in such a way that promotes tumor-specific T-cell mediated killing of melanoma cells (9).

Except for gp100, TAP-1 and TAP-2 have an important role in the recognition of melanoma cells by CD8 CTLs, as they transport peptides processed by the proteasome into the endoplasmic reticulum, where they are loaded onto MHC class II (11). Setiadi et al. showed that HDACI are able to increase the expression of TAP-1 by enhancing recruitment of RNA polymerase II to the TAP promoter so as to promote immune recognition of melanoma (11). Cao et al. showed that HDACI have the possibility to increase the action of the immune system by preventing the activation-induced cell death. In this study TSA was approved, the first FDAapproved HDACI that causes down-regulation of FasL expression on infiltrating $\mathrm{CD}^{+} \mathrm{T}$ cells, to inhibit the apoptosis of $\mathrm{CD}^{+} \mathrm{T}$ lymphocytes. Additionally, the same study showed that TSA and CTLA4 antibody act synergistically to enhance $\mathrm{CD} 4^{+} \mathrm{T}$-cell infiltration and that TSA can increase the expression of Il-2, TNF-a and GHCSF, cytokines associated with cell activation (23).

It is known that HDACI have a great anti-proliferative effect on melanoma cells $(6,9,10,12)$. Borrielo et al. demonstrated that HDACI of class I and II can cause the accumulation of melanoma cells in G1 phase due to the elongation of p27half-life. The p27 is a CDKI, which can mediate $G_{1}$ arrest in response to an array of stimuli including DNA damage, mitogen deprivation or drug treatment. The level of this protein can be decreased by ubiquitination followed by proteasomal degradation. HDACI have the capability to down-regulate Skp2 protein, a component of the nuclear p27 ubiquitination complex due to translational and post translational mechanism to increase p27half-life (12).

Most HDACI can induce the expression of cyclindependent kinase inhibitors (CK1) p21 by increasing acetylation of the chromatin at the sp1 binding site in the promoter region of the gene. High levels of p21 are associated with $\mathrm{G} 1 / \mathrm{S}$ phase cell cycle arrest due to the inhibition of CDK2 $(10,29)$.

Additionally, HDACI can cause down-regulation of $\mathrm{TbX} 2$, a protein that represses $\mathrm{p} 21$ expression in vitro and in vivo and plays an important role in melanoma proliferation $(10,29)$. Vance et al. showed that $\mathrm{TbX} 2$ is over-expressed in melanoma cells and that HDAC proteins are potential mediators of $\mathrm{TbX} 2$ proteins, a fact that can explain the capability of HDACI to cause down-regulation of $\operatorname{TbX} 2(10,29,30)$.
HDACI can also induce autophagy in melanoma cells through FOXO1-dependent pathways. Forkhead box proteins (FOXOs) are transcription factors which have an important role in regulation of genes involved in cell growth, proliferation and differentiation, with FOXO1 being the most studied (Autophagy is a mechanism which provides cells with additional nutrient supplies under stress conditions and is mediated by the suppression of rapamycin, MTOR, a negative regulator of autophagy) $(22,31)$. Zhang et al. approved that HDACI can enhance FOXO1 acetylation and transcriptional activation by suppressing the action of deacetylases. This way the transcription of ATGs genes increases (autophagy related genes), which are necessary for the autophagy of cancer cells (22).

Nowadays, several HDACI such as vorinostat, entinostat and valproic acid have recently been tested in phase I and early phase II trials, yet most agents show limited efficacy and tolerability as single agents. Haematological toxicity, fatigue, nausea and laboratory abnormalities are the most frequent adverse events of HDAC inhibition $(24,32)$.

\section{Conclusion}

In recent years many studies have focused on examining the efficacy of HDACI in the treatment of melanoma, with TSA being the most well studied. It has been approved that HDACI play an important role in the restriction of the growth and proliferation of melanoma cells especially by inducing cell cycle arrest and apoptosis with different mechanisms. In addition, the synergistic action of HDACI with other inhibitors such as BRAF inhibitors and BET inhibitors has been studied and has been approved as a quite effective treatment of melanoma but further research is required on this subject. The fact that four HDACI, vorinostat (5AIIA, Zalmza), romidepsine (Istodox, depsipeptide), belinostat and panobinostat (Farydak) have been accepted by the FDA for treatment of CTCL (cutaneous T-cell lymphoma) and PTCL (peripheral T-cell lymphoma) and multiple myeloma means that HDACI are molecules which will be used further in the future for cancer therapy and more research may result in the acceptance of these molecules by the FDA for melanoma treatment.

\section{References}

1 Chodurek E, Kulczycka A, Orchel A, Aleksander-Konert E and Dzierzewicz Z: Effect of valproic acid on the proliferation and apoptosis of the human melanoma G-361 cell line. Acta Pol Pharm 71: 917-921, 2014.

2 Chodurek E, Orchel A, Gawlik N, Kulczycka A, Gruchlik A and Dzierzewicz Z: Proliferation and cellular death of A375 cell line in the presence of HDACs inhibitors. Acta Pol Pharm 67: 686689, 2010. 
3 Shan X, Fu YS, Aziz F, Wang XQ, Yan Q and Liu JW: Ginsenoside $\operatorname{Rg} 3$ inhibits melanoma cell proliferation through down-regulation of histone deacetylase 3 (HDAC3) and increase of p53 acetylation. PLoS One 9: e115401, 2014.

4 Gatti L, Sevko A, De Cesare M, Arrighetti N, Manenti G, Ciusani E Verderio P, Ciniselli CM, Cominetti D, Carenini N, Corna E, Zaffaroni N, Rodolfo M, Rivoltini L, Umansky V and Perego P: Histone deacetylase inhibitor-temozolomide cotreatment inhibits melanoma growth through suppression of Chemokine (C-C motif) ligand 2-driven signals. Oncotarget 5: 4516-4528, 2014.

5 Gallagher SJ, Tiffen JC and Hersey P: Histone Modifications, Modifiers and Readers in Melanoma Resistance to Targeted and Immune Therapy. Cancers (Basel) 7: 1959-1982, 2015

6 Damaskos C, Karatzas T, Nikolidakis L, Kostakis ID, Karamaroudis S, Boutsikos G, Damaskou Z, Kostakis A and Kouraklis G: Histone Deacetylase (HDAC) Inhibitors: Current Evidence for Therapeutic Activities in Pancreatic Cancer. Anticancer Res 35: 3129-3135, 2015.

7 Lai F, Jin L, Gallagher S, Mijatov B, Zhang XD and Hersey P: Histone deacetylases (HDACs) as mediators of resistance to apoptosis in melanoma and as targets for combination therapy with selective BRAF inhibitors. Adv Pharmacol 65: 27-43, 2012.

8 Heinemann A, Cullinane C, De Paoli-Iseppi R, Wilmott JS, Gunatilake D, Madore J, Strbenac D, Yang JY, Gowrishankar K, Tiffen JC, Prinjha RK, Smithers N, McArthur GA, Hersey P and Gallagher SJ: Combining BET and HDAC inhibitors synergistically induces apoptosis of melanoma and suppresses AKT and YAP signaling. Oncotarget 6: 21507-21521, 2015.

9 Murakami T, Sato A, Chun NA, Hara M, Naito Y, Kobayashi Y, Kano Y, Ohtsuki M, Furukawa $Y$ and Kobayashi E: Transcriptional modulation using HDACi depsipeptide promotes immune cell-mediated tumor destruction of murine B16 melanoma. J Invest Dermatol 128: 1506-1516, 2008.

10 Boyle GM, Martyn AC and Parsons PG: Histone deacetylase inhibitors and malignant melanoma. Pigment Cell Res 18: 160$166,2005$.

11 Setiadi AF, Omilusik K, David MD, Seipp RP, Hartikainen J, Gopaul R, Choi KB and Jefferies WA: Epigenetic enhancement of antigen processing and presentation promotes immune recognition of tumors. Cancer Res 68: 9601-9607, 2008.

12 Borriello A, Naviglio S, Bencivenga D, Caldarelli I, Tramontano A, Speranza MC, Stampone E, Sapio L, Negri A, Oliva A, Sinisi AA, Spina A and Della Ragione F: Histone deacetylase inhibitors increase $\mathrm{p} 27^{\mathrm{Kip} 1}$ by affecting its ubiquitin dependent degradation through Skp2 down-regulation. Oxid Med Cell Longev 2016: 2481865, 2016.

13 Woods DM, Woan K, Cheng F, Wang H, Perez-Villarroel P, Lee C, Lienlaf M, Atadja P, Seto E, Weber J, Sotomayor EM and Villagra A: The antimelanoma activity of the histone deacetylase inhibitor panobinostat (LBH589) is mediated by direct tumor cytotoxicity and increased tumor immunogenicity. Melanoma Res 23: 341-348, 2013.

14 Kato Y, Salumbides BC, Wang XF, Qian DZ, Williams S, Wei Y, Sanni TB, Atadja P and Pili R: Antitumor effect of the histone deacetylase inhibitor LAQ824 in combination with 13-cisretinoic acid in human malignant melanoma. Mol Cancer Ther 6: 70-81, 2007.

15 Millward M, Price T, Townsend A, Sweeney C, Spencer A, Sukumaran S, Longenecker A, Lee L, Lay A, Sharma G,
Gemmill RM, Drabkin HA, Lloyd GK, Neuteboom ST, McConkey DJ, Palladino MA and Spear MA: Phase 1 clinical trial of the novel proteasome inhibitor marizomib with the histone deacetylase inhibitor vorinostat in patients with melanoma, pancreatic and lung cancer based on in vitro assessments of the combination. Invest New Drugs 30: 23032317, 2012.

16 Namdar M, Perez G, Ngo L and Marks PA: Selective inhibition of histone deacetylase 6 (HDAC6) induces DNA damage and sensitizes transformed cells to anticancer agents. Proc Natl Acad Sci USA 107: 20003-20008, 2010.

17 Wilking MJ, Singh CK, Nihal M, Ndiaye MA and Ahmad N: Sirtuin deacetylases: a new target for melanoma management. Cell Cycle 13: 2821-2826, 2014.

18 Zhang XD, Gillespie SK, Borrow JM and Hersey P: The Histone deacetylase inhibitor suberic bishydroxamate regulates the expression of multiple apoptotic mediators and induces mitochondria dependent apoptosis of melanoma cells. Mol Cancer Ther 3: 425-435, 2004.

19 Gillespie S, Borrow J, Zhang XD and Hersey P: Bim plays a crucial role in synergistic induction of apoptosis by the histone deacetylase inhibitor SBHA and TRAIL in melanoma cells. Apoptosis 11: 2251-2265, 2006.

20 Klisovic DD, Katz SE, Effron D, Klisovic MI, Wickham J, Parthun MR, Guimond M and Marcucci G: Depsipeptide (FR901228) inhibits proliferation and induces apoptosis in primary and metastatic human uveal melanoma cell lines. Invest Ophthalmol Vis Sci 44: 2390-2398, 2003.

21 Greer CB, Tanaka Y, Kim YJ, Xie P, Zhang MQ, Park IH and Kim TH: Histone deacetylases positively regulate transcription through the elongation machinery. Cell Rep 13: 1444-1455, 2015.

22 Zhang J, Ng S, Wang J, Zhou J, Tan SH, Yang N, Lin Q, Xia D and Shen HM: Histone deacetylase inhibitors induce autophagy through FOXO1-dependent pathways. Autophagy 11: 629-642, 2015.

23 Cao K, Wang G, Li W, Zhang L, Wang R, Huang Y, Du L, Jiang J, Wu C, He X, Roberts A, Li F, Rabson AB, Wang Y and Shi Y: Histone deacetylase inhibitors prevent activation-induced cell death and promote anti-tumor immunity. Oncogene 34: 59605970, 2015 .

24 Hornig E, Heppt MV, Graf SA, Ruzicka T and Berking C: Inhibition of histone deacetylases in melanoma-a perspective from bench to bedside. Exp Dermatol 25: 831-838, 2016.

$25 \mathrm{Li} \mathrm{T}$, Song B, Wu Z, Lu M and Zhu WG: Systematic identification of Class I HDAC substrates. Brief Bioinform 15: 963-972, 2014.

26 Krumm A, Barckhausen C, Kücük P, Tomaszowski KH, Loquai C, Fahrer J, Krämer OH, Kaina B and Roos WP: Enhanced histone deacetylase activity in malignant melanoma provokes RAD51 and FANCD2-triggered drug resistance. Cancer Res 76: 3067-3077, 2016.

27 West AC, Smyth MJ and Johnstone RW: The anticancer effects of HDAC inhibitors require the immune system. Oncoimmunology 3: e27414, 2014.

28 Rocca A, Minucci S, Tosti G, Croci D, Contegno F, Ballarini M, Nolè F, Munzone E, Salmaggi A, Goldhirsch A, Pelicci PG and Testori A: A phase I-II study of the histone deacetylase inhibitor valproic acid plus chemoimmunotherapy in patients with advanced melanoma. Br J Cancer 100: 28-36, 2009. 
29 Vance KW, Carreira S, Brosch G and Goding CR: Tbx2 is overexpressed and plays an important role in maintaining proliferation and suppression of senescence in melanomas. Cancer Res 65: 2260-2268, 2005.

30 González-Pardo V, Suares A, Verstuyf A, De Clercq P, Boland $\mathrm{R}$ and de Boland AR: Cell cycle arrest and apoptosis induced by $1 \alpha, 25(\mathrm{OH}) 2 \mathrm{D} 3$ and TX 527 in Kaposi sarcoma is VDR dependent. J Steroid Biochem Mol Biol 144: 197-200, 2014.

31 Kim E, Bisson WH, Löhr CV, Williams DE, Ho E, Dashwood RH and Rajendran P: Histone and Non-Histone Targets of Dietary Deacetylase Inhibitors. Curr Top Med Chem 16: 714$731,2016$.
32 Díaz-Núñez M, Díez-Torre A, De Wever O, Andrade R, Arluzea J, Silió M and Aréchaga J: Histone deacetylase inhibitors induce invasion of human melanoma cells in vitro via differential regulation of Ncadherin expression and RhoA activity. BMC Cancer 16: 667, 2016.

Received July 23, 2017

Revised August 2, 2017

Accepted August 4, 2017 